\title{
Yasmine's Adventures: An interactive urban experience exploring the sociocultural potential of digital entertainment
}

\author{
Valentina Nisi ${ }^{1}$, Mara Dionisio ${ }^{1}$, Julian Hanna ${ }^{1}$, Luis Ferreira ${ }^{1}$, Nuno Nunes ${ }^{1}$ \\ ${ }^{1}$ Madeira-ITI, University of Madeira. Campus da Penteada 9020-105, Funchal, Portugal \\ \{mara.dionisio, julian.hanna, luis.ferreira\}@m-iti.org \\ $\{$ njn, valentina\}@uma.pt
}

\begin{abstract}
Urban computing systems impact quality of life in densely populated areas. With the widespread availability of wireless networks and portable devices, urban areas are fast becoming a hybrid of the physical environment and the digital datasphere. This paper describes Yasmine's Adventures, a location aware storytelling platform that leverages on urban computing strategies to create an interactive walk through the Mehringplatz area, surrounding the Jewish Museum in Berlin. Yasmine's Adventures (YA) is a mobile application that delivers a sequence of animations clips tailored specifically to the Mehringplatz neighbourhood. The story follows an adventurous local girl as she walks home alone, visiting local landmarks. Yasmine's perceptions of the landmarks, identified by community members in an earlier workshop, reflect the real concerns of the community. This interactive experience was created to engage visitors of the Jewish Museum to explore the relatively neglected streets of the area in which the museum is situated.
\end{abstract}

Keywords: Mobile socially driven entertainment, Interactive narrative, Digital storytelling, Location aware virtual reality, Urban computing

\section{$1 \quad$ Introduction}

Urban computing systems [1] can have a powerful impact on improving quality of life in densely populated areas. With the widespread availability of wireless networks and portable computing devices urban areas are fast becoming a hybrid of the physical environment and the digital datasphere. Our work falls into an area known as 'locative media' [26], which frames mobility not as a problem to be overcome but as both an everyday fact and a new opportunity to create interactive experiences that rely upon or exploit movement and space. The motivation for this work is that the conscious layering of space and narrative provides a deeply compelling experience and a high level of immersion [25]. Moreover, understanding mobility in its cultural settings requires that we pay attention to the symbolic and aesthetic aspects of technological urbanism as well as the purely instrumental [19].

This paper describes Yasmine's Adventures, a location aware storytelling platform that leverages on urban computing strategies to create an interactive trail across the 
landscape surrounding Mehringplatz in the Friedrichshain-Kreuzberg borough of Berlin. This interactive walk was created with the goal of challenging and engaging visitors of the world famous Jewish Museum Berlin to explore the adjacent and relatively neglected streets of the area in which it is situated.

Historically (until the 18th century) one of the three major squares of the city, Mehringplatz today is quite a different place. Located in central Berlin, today the area is considered by many to be unremarkable at best, ugly and unfriendly at worst. Marked as a socially disadvantaged neighbourhood, it has acquired a negative reputation in recent decades. In contrast, the Jewish Museum Berlin, built by architect Daniel Libeskind in 2001, is an award-winning, internationally recognized building. Local residents of Mehringplatz have been actively trying for years to change the area's reputation, for example by emphasizing its multicultural identity, its tolerance and diversity, and its welcoming attitude. Such qualities are often overlooked by visitors to the area, however, who are usually focused on visiting the museum. Leveraging on the colocation of the Jewish Museum, which attracts more than 700,000 visitors per year [2], we took the opportunity to shine a spotlight on the Mehringplatz community by revealing the everyday lives, local landmarks, and important issues of the community to visitors of the museum. Through our interactive walk we created an opportunity for people to wander the local streets and establish a link between the museum and the neighbourhood in which it is embedded.

Through the interactive walk the audience follows the adventures of a free-spirited local girl named Yasmine, as she sneaks away from her class field trip to the museum and attempts to walk home alone. The story is delivered through the Yasmine's Adventures (YA), a mobile application that delivers a sequence of short animations tailored specifically to the Mehringplatz neighbourhood: revealing, through the adventures of Yasmine, the community's feelings about itself. The 2D animated stories are embedded in 3D virtual environments that reproduce the real features of the Mehringplatz streets. These 3D panoramas are revealed to users when they capture specific markers (See Fig. 3 below) strategically positioned in order to lead the audience from the Jewish Museum into the heart of Mehringplatz.

The locations where each segment of Yasmine's Adventures takes place were determined by community members as part of the RDL/UdK-led 'Pinpointing Mehringplatz' workshop [3]. Each pinpointed place holds specific positive or negative values for the local residents. By seeing the neighbourhood through Yasmine's eyes, the audience also sees it through the eyes of the community: the most beloved spaces, the areas that require change, and the spaces that are disliked or seen as problematic by the community all feature in Yasmine's story.

The Yasmine's Adventures project was developed to be showcased at the international symposium Community Now? The Politics of Participatory Design, which took place from 19-21 February at the Jewish Museum Berlin. 


\section{Related Work}

Urban computing is generally defined as the process of acquiring, integrating, and analyzing big and heterogeneous data generated by diverse sources in urban spaces, such as sensors, devices, vehicles, buildings, and humans [20]. Urban computing investigates the ways in which computing technologies shape, are shaped by, and mediate our experience of urban space, reflecting the contemporary reality of the city as a nexus of computational infrastructures [19]. The areas of research in mobility and mobile computing applications are driven by two primary considerations: i) mobilizing static applications allowing people to carry on traditional desktop tasks while on the move; and ii) providing people with access to resources in unfamiliar spaces, helping people navigate space in terms of resources such as devices, services, or people [19]. In this context patterns of movement go beyond moving from point $\mathrm{A}$ to $\mathrm{B}$ and may enact social and cultural meanings; therefore navigation through urban space enacts aspects of cultural identity. Such work falls under location-based social networks in which individuals are connected by interdependency derived from their locations in the physical world as well as their location-tagged media content [20].

Creative interventions in this domain fall under locative media in which location and time are considered essential to the work [24], alluding to the fact that a user's context and their movement through space need to be taken into consideration during the design process for a mobile media system. Locative systems encompass a number of different fields and applications, from art to academic research, including many different authoring tools, games and narrative experiences [8]. Location Aware Multimedia Stories (LAMS) is one area of locative media. LAMS refers to cinematically rendered narrative content related to specific locations and embedded in those real spaces through the use of location aware mobile technologies [8]. LAMS combine the mobility of the audience with spatial distribution of the story content in interactive, multi-threaded narrative experiences to create a synergy that encourages the development of a sense of place from otherwise unknown spaces.

Urban spaces are rich and multilayered in symbolic and material meanings [4]. Complex layers of history alter their physical and social aspect. Today, mobile devices and the consumption of mobile multimedia are ubiquitous. By designing and producing virtual layers of information that combine with the existing material layers we can have a direct impact on how a location is perceived and experienced by visitors and residents. Nowadays cities are often augmented with different layers of multimedia content in order to tailor specific experiences for their users. Several projects in the past two decades have explored the association of digital media to urban locations with the intention of providing rich entertaining and educating experiences $[5,6]$. These projects have in effect laid the foundations and established guidelines for how to design mobile location aware experiences. In our case, we learned from these projects how to carefully orchestrate a complete mobile experience, pacing content to avoid overloading the audience with overlapping tasks and media. In particular a number of these projects focused specifically on empowering communities, highlighting local histories $[7,8,9]$, counteracting media reports that damaged the reputations of disadvantaged neighbourhoods [10], and connecting communities with visitors [11]. 
Building on this work, we envisaged leveraging on past findings while tackling some of the challenges that still remain unresolved. For example, how to address GPS inaccuracy in pinpointing exact locations, or how to best combine and take advantage of different location aware sensors and technologies such as iButtons, RFID tags, or QR-Codes to refine the location specificity of the application $[12,13,14]$. Moreover, even when the technical layer of the design seems resolved, practical issues still crop up: for example, how to ensure that tags or other equipment left outdoors are not vandalized, how obtain permissions for working in public spaces, or how to reflect the specific (e.g. socio-economic) realities of a location while guaranteeing user safety.

To address some of the above issues many projects resort to virtual reality (VR) and augmented reality (AR) as means to associate content to locations. Such technologies can serve as a highly efficient means of anchoring the viewer to the real space around them [15]. However the low accuracy and frequency of AR can create jerky augmentation, which may negatively impact the user experience [16, 17]. For this reason many projects opt instead for virtual reality, which means reconstructing the environment virtually and overlaying it with content inside the $3 \mathrm{D}$ reconstruction. For example, in the project 'Matera: Tales of a City', the authors used virtual reality environments to share memories and stories that took place in that ancient physical location. This approach, in addition to site-specific viewing, supports location independent viewing, where the content can be uploaded to the Internet for later reflection and recollection [18]. In this way the project expands its potential audience and the timeframe in which the experience can be enjoyed by the public.

In summary, several projects over the last two decades have explored the association of digital media to urban locations with the intent of providing rich experiences that take advantage of urban technologies. The related work analyzed above has informed some of our design decisions, described in detail in the section below.

\section{Designing Yasmine's Adventures Interactive Experience}

\subsection{The Concept}

Yasmine's Adventures was conceived with the goal of exposing non-residents (in this case the symposium audience) to the issues and attractions of the Mehringplatz neighbourhood by encouraging them to see the area from the point of view of the community. The process originated with the 'Pinpointing Mehringplatz' workshops, in which local community members expressed opinions about features of the neighbourhood as pleasing, displeasing, or potentially transformational. Locations were chosen and photographed by community members themselves, led by UDK.

By analyzing the locations and content provided to us by UDK workshop organizers, we identified a convenient route leading participants from the museum into the heart of the neighbourhood. This route took into consideration the constraints of a frigid Berlin winter, hence not exposing people to harsh weather conditions for more than 20 minutes, after which any experience would become unpleasant. Based on the issues raised by the community members themselves we selected five anchor points for our interactive walk. Some had positive connotations, like the local hip hop youth 
space or murals that had been painted by famous artists. Other locations had negative connotations for the community, like the construction site at the center of the neighbourhood and the dirty alleys behind some of the apartments. Yasmine's Adventures echoes the positive or negative feelings about neighbourhood locations. For example, Yasmine is scared and unhappy near the construction site, because of its ugly intrusion into the landscape. Conversely she has fun at the youth club dancing to hip hop. Yasmine's Adventures highlights the community's concerns, making them visible outside the community.

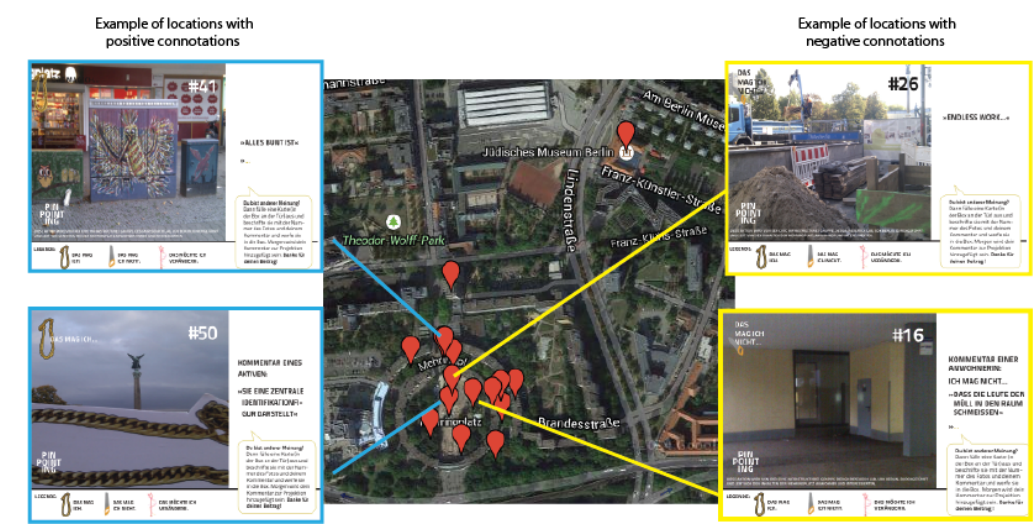

Fig. 1. Map of the neighbourhood with all 'Pinpointing Mehringplatz' locations. On the left and right are samples of content produced by the community in the original format.

\subsection{The Story}

In order to engage our audience in following someone through the neighbourhood, we wanted a character who would elicit empathy. For this reason we opted for a child protagonist and created Yasmine, a 7-year-old girl from the neighbourhood. Her appearance reflects the ethnic diversity of Mehringplatz. She is a curious and adventurous girl. The story starts with her school trip to the Jewish Museum, where our interactive walk was made available to the public. Yasmine is bored by the strict rules of the school trip and decides to escape, running away from the museum and walking home on her own. Along the journey she stops in various places such as the children's playground and the KMA where hip hop classes are offered (both very positive). Continuing her journey, she follows a painter to the popular street murals. She talks to the painter and learns about the murals. Then the story moves into a fantastic event, as one of the murals comes to life and a big bird depicted in it flaps its wings and flies into the air. Yasmine decides to follow the bird as it flies away, finally landing on the angel statue at the center of the square. Near the angel is where much of the unpopular construction is taking place: a location that the community would like to change. Here Yasmine starts to become worried: it is getting dark and the construction site is scary. She runs off and then, tired and scared, declares that she wants to go home. But one last obstacle must be overcome: she finds herself in an alley near the apartment block 
where she lives, a site that the community labelled as dirty and often full of rats. As the night turns stormy, a flash of lightning illuminates the alley, and she sees the menacing shadow of a rat. She remembers something given to her by the angel, a magic feather, and that helps her to find the way home.

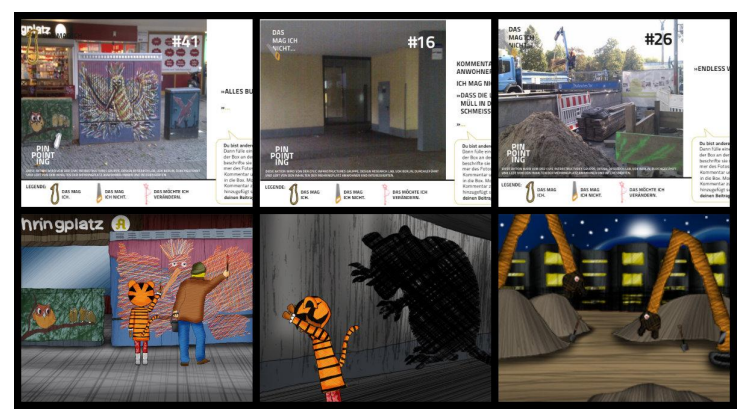

Fig. 2. Connection between community content and Yasmine's story. Left to right: the first top and bottom pictures show the mural and Yasmine learning about murals in Mehringplatz by talking to the painter. Second top and bottom: the dirty alley as reported by the community, where Yasmine meets the rat. Last top and bottom: the construction site in Mehringplatz.

\subsection{The Experience Design}

Ideally our audience would become aware of the interactive walk at the Jewish Museum through the ticket desk or posters. If interested they would be given a phone or instructed how to download the application to their own device and how to use the app. The user then has to go outside the museum and look for visual cues or markers. These markers, located in strategic spots, are easy to see and access. The markers are A5 postcards depicting scenes from Yasmine's Adventures. By pointing the phone's camera at the marker and capturing it, a 3D reconstruction of the surrounding environment loads and is displayed on the screen. Once the 3D environment appears the user is prompted to scan the real environment looking for story content. Thanks to the phone's accelerometer and compass, the 3D reconstruction of the environment follows the user's movements, updating the screen with a 3D version of the real environment outside. Within the 3D environment story elements are highlighted by an orange circle. The orange circle is a loading cue: it means that story content related to that precise spot in that location is loading. The user stops and waits until the video animation is fully loaded and then watches it. The animation depicts adventures in that specific location.

When the video is over, the user returns to the $3 \mathrm{D}$ environment screen by clicking a back button, and scans the real environment for more stories. If no more stories are available, the user can go back to the 2D map and follow the indications of where to go next to see more adventures of Yasmine. The application covers content in six different locations, all reconstructed in 3D. One or more 2D animations of Yasmine's Adventures are found in each location. The story is sequential so the locations have to be visited in the correct order. Yasmine's story ends after all six locations highlighted 
on the map have been visited. The user then returns the device. The walk lasts approximately 20 minutes: the duration was a design consideration due to the cold Berlin winter, which could lead to an unpleasant or unfinished experience.

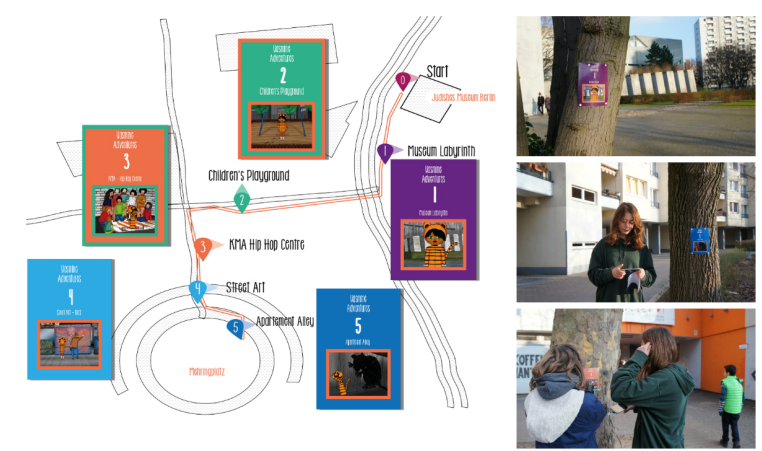

Fig. 3. Left: The experience points with content associated to the visual marker. Right: The visual markers placed in the real environment and participants interacting with them.

\subsection{Technical description}

The implementation of the mobile application that delivers the Yasmine's Adventures Experience was programmed in $\mathrm{C} \#$ using the Unity ${ }^{1}$ game engine. The main interface is composed of a scrollable map, a 'close' button, and a 'capture the marker' button (see Fig. 4). In order to recognize and capture the visual markers we used Vuforia, third party software developed by Qualcomm ${ }^{2}$ and available as a plugin for Unity. Vuforia is a mobile vision platform offering many features to generate vision-based computing experiences. Vuforia allowed us to associate a 'visual marker' with specific content, in our case a 360-degree 3D panorama. The use of this plugin allows us total freedom in the design of the 'visual marker' since it detects and tracks features that are naturally found in the image itself and compares them with a known target resource database ${ }^{3}$.

Each marker is associated with a 360 -degree 3D virtual environment. To achieve the 360-degree interaction we used the Durovis Dive plugin developed by Durovis ${ }^{4}$ expressly for Unity. Durovis Dive transforms a mobile device into a virtual reality headset using a nylon viewer that features two adjustable lenses. These lenses project an image of the virtual scene into each eye. This feature is combined with headtracking software that makes use of the mobile device's inbuilt gyroscope. For our purposes we tweaked the software so that we did not have to use the viewer for the $3 \mathrm{D}$ effect, and only required the virtual scene to display on the mobile device. For this

\footnotetext{
http://unity3d.com/

https://www.qualcomm.com/products/vuforia

https://developer.vuforia.com/library/articles/Training/Image-Target-Guide

4 http://www.durovis.com
} 
we were able to implement an interactive 360-degree virtual environment playing from a Samsung Galaxy S4 mobile device.

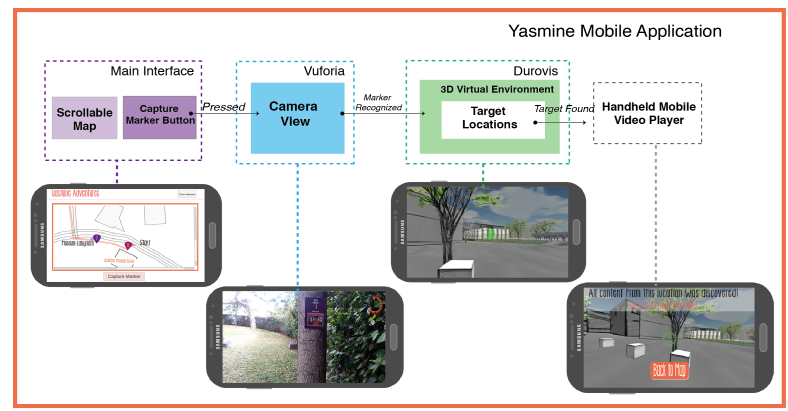

Fig. 4. Yasmine mobile application flow. Top: The flow of the technical implementation can be seen. Bottom: Corresponding screenshots.

For YA we recreated five three dimensional virtual locations of the neighbourhood in which to disseminate the animations. The 3D models had to be low poly to reduce the processing power needed to render them. The real locations chosen were modeled and textured according to the visual style of the animation (see Fig. 5). The entire surrounding environment in the locations is rendered in shades of grey except for the exact location where the story happens. This way the colored features of the environment attract the attention of the user to the content. Once the target location in the virtual environment is found and users lock their scanning movement on it, the animation video loads and plays. When the video playback finishes Unity resumes, showing if there is more content to explore or not. All the multimedia content is stored on the mobile device and no data connection is needed.

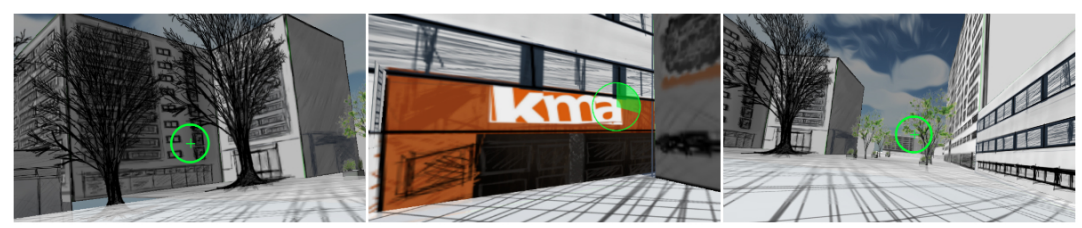

Fig. 5. Screenshoot of sections of the 3D panoramas at the KMA location. The green circle indicates content presence and loading(central picture)

\subsection{Mixing Realities}

While designing the Yasmine's Adventures Experience we had to make several design and technology choices. In the following section we will describe our design rationale in detail. In order to be able to highlight the precise connection of the content to specific points on the location, we opted for reconstructing the environment surrounding the users as a 3D environment. In fact replicating the real environment surrounding the user on the device and placing content in the $3 \mathrm{D}$ environment itself allowed us unprecedented precision in communicating to the player the relationship of 
the content to that exact space. GPS alone would not have given us precise support for the connection between the stories of Yasmine and the places pinpointed by the community. At the same time, the real space around the user still acts as reference, as the $3 \mathrm{D}$ world depicts the same environment.

We decided to adopt physical visual markers to indicate the presence of content around the neighbourhood streets. Adopting markers in order to trigger the related 3D reconstruction of the surrounding environment allowed us to place the markers in the vicinity but still in a safe and accessible place for the player to stop, capture and view the content. Once the marker is captured, the user can move from the place where the marker was positioned and still carry the reference to the content in the $3 \mathrm{D}$ environment of the device. Through the act of scanning the 360-degree environment the user becomes familiar with the surrounding peripheral space. S/he can then lock the motion in the direction of the related content, which is the same precise spot in the 3D environment. After viewing the content the user can resume looking around and checking the real surrounding place to reflect on what s/he just saw. The replication of real space in 3D also evokes a sense of wonder and contrast between real and virtual. Through this design we wanted to open up space for imagination, assisting the viewer in thinking about the space in different ways (e.g. from the point of view of a neighbourhood child), or deepening immersion in the story itself.

As the project has a strong community focus, while designing the application we envisaged the eventual possibility of community members and visitors adding more stories or comments to the highlighted locations, as well as adding new ones. We foresaw the experience in later iterations fostering dialogue and eliciting ideas and comments from residents and non-residents alike. Such changes would add a layer of feedback and participation of the user and eventually of the community itself. The details of such an extension of the system have not yet been designed or discussed in detail, belonging to the possible future work and directions of the project.

Furthermore, we envisaged the experience as contained in the 3D panoramas and animations available for sharing with non collocated audience via the Internet. In this way it would be available to appeal to a larger audience without losing the site specificity of the story, which would remain captured in the 3D reconstructions of the environment. A sort of virtual representation of Mehringplatz and the issues highlighted by its own community would be created, sharable and updatable through the Web.

\subsection{Pilot and findings}

We piloted the YA application during the Community Now? symposium, which took place at the Jewish Museum Berlin in February 2015. The aim of the pilot was to collect feedback for further refinements of the application and guidance for the design of a wider user study. The walk was available to symposium guests during the lunch break. A special desk was set up just outside the symposium lecture room with three researchers and ten mobile phones available for guests to borrow. Participants borrowed the mobile phones with the interactive application already installed. The researchers at the desk explained how to use and interact with the application, how to find and capture the markers along the way, and where to return the devices at the end 
of the experience. After the explanation two of the researchers followed the users as they interacted with the story, shadowing them and observing their actions. The users were told that the researchers would be around and available to help and answer questions if they encountered any problems. At the end of the experience there was a onepage questionnaire for users to complete with details of their experience. Because we were testing an experience we decided to focus on flow [21]. Flow is a subjective state in which the person is intensely involved in a task, ignoring other stimuli and being fully invested in a precise challenging but achievable activity [21]. Flow theory has been applied in many different domains of experience, including sport, art, work, games, and HCI [22]. The questionnaire includes some demographics and a section intended to evaluate the flow of the experience through several Likert scale questions. Flow is a fairly stable concept, but the techniques used to measure flow vary significantly. For the purpose of our pilot evaluation we used a simplified version of the flow questionnaire described in [23]. The questionnaire also included space to write down free comments for qualitative assessment of the experience.

Several participants took the walk and communicated reactions to the researchers in person, while four completed the flow questionnaire. The pilot was a limited qualitative probe into the experience, in order to gain insights and lay groundwork for the design of a wider study. Pilot findings are described in detail in the following section. The results involve the analysis of the feedback gathered through observations, questionnaires, written comments, and loose feedback during the experience and the rest of the symposium day.

Flow of the experience: Flow experience is achieved in conjunction with activities that are simultaneously and equally providing fluency (e.g. 'I knew what I had to do each step of the way', 'I felt that I had everything under control') and a high level of absorption (e.g. 'I didn't notice time passing'). From the four completed questionnaires the results are promising. All users indicated high levels for losing the notion of time and medium-high levels for being absorbed in what they were doing. The degree of challenge was appropriate despite the fact that most users had low familiarity with this kind of interactive experience. In terms of fluency, users reported that they did not have difficulty concentrating and felt like they had everything under control, which indicates some fluency. Although the sample is not significant these initial results are encouraging in indicating the presence of a flow state while participating in the experience.

Orienting the user in finding and viewing the content: From the voiced and written comments of the users and the observations of researchers on the ground it was clear that people were having difficulty identifying the first marker. One user in particular mentioned that the term 'visual marker' invited him to expect a marker similar to a QR code. In order to mitigate the problem, suggestions ranged from using a different name for the marker, to making an introductory video. The video would clarify details of the experience and explain how the system worked so users would not have to rely on a human guide to explain to them how to take the tour. 
Moreover, the indicator of presence and loading of video content in the 3D panorama (the orange circle) was flagged as difficult to spot. Researchers on the ground had to help users find the indicator on the screen and ask them to wait until it indicated full loading before moving away from that spot. As a result, some people did not always find the video content inside the 3D panorama and would move to the next location, missing out on a sequential fragment of the story. Some users who did spot the orange indicator did not realize that the content was loading (slowly) and would move to the next location too quickly, losing partially loaded content and missing out on the story.

Closure for the experience: Some users reported that the experience somehow lacked closure: 'I felt like I needed a conclusion at the end of the experience explaining more details' (U4). In addition, the practical task of returning the devices was not streamlined properly. Many users went for lunch and kept the device with them, intending to return it later. This problem was partly caused by the fact that participants had lunch near the location of the last story, and we did not enforce bringing back the device since the weather was cold and people were hungry. But the outcome of the pilot made us reflect on how devices could be returned without the users having to going back to the museum.

Comments from the questionnaire: Three users explicitly reported enjoying the experience, in particular the graphics, layout, and interface: 'It was fun! I liked the interface and the layout' (U2); 'very inspiring' (U3). Some users suggested the experience would be inspiring as a community tool for sharing opinions about their neighbourhood, as well as to entice visitors to engage with an area, although it needed refinements. One user praised the connection of geocaching with social content from the community: 'I liked the idea of combining geocaching and socio-cultural information.' The same user highlighted how such a method fostered deeper engagement and exploration of the locations. Users reported that they saw things in the neighbourhood they would have not otherwise, such as the murals: 'It's a great way to look in detail at certain points' (U3).

\section{Discussion and Future work:}

Our preliminary evaluation of the YA provided useful insights for improving the interactive experience. In this section we describe the changes introduced after the pilot.

We created an introductory video with information on how to use the mobile application, its purpose, and the overall experience. In this video we replaced the problematic 'marker' term with the more descriptive 'postcard'. The orange circle indicating content and loading of the animation file was changed to a bright green which was much more visible than the previous. Some additional explanatory text was added beside the green circle in order to alert users to wait until the loading of the animation was finished. We added text to $3 \mathrm{D}$ environments containing more than one animated 
story to tell users to continue searching for stories after the first had been viewed. Refinements to the story clips were made to add context and make some stories more complete: for example, an extra scene at the beginning of the first story showing the children getting off the school bus in front of the Jewish Museum.

Details were added to the landscape to improve aesthetics and user engagement: for example, more leaves on the trees and hedges, a harder rain during one of the dramatic scenes, and more detail in the characters' expressions. Extra dialogue between Yasmine and the locals was added so that more information about the neighbourhood could be conveyed to the audience. A final epilogue clip was also made in order to wrap up the experience in a more satisfying way. In the last clip the links between Yasmine's Adventures and the issues highlighted by the community during the workshop were explained in detail. Details about returning the device were also added to the epilogue.

Pilot testing of the YA guided the design and deployment of a wider evaluation of the mobile urban experience. On the basis of the pilot experience and feedback received we designed a more detailed questionnaire probing the audience on immersion and flow aspects of the experience as well as their level of engagement with the neighbourhood. The evaluation was carried out in the same location one month after the symposium. We managed to recruit 20 users and engaged them in a 20 -minute walk with the YA system, a 15-minute exit interview, and a 10-minute questionnaire. Preliminary findings support the assumption that the application promotes relatedness and exploration of the local neighborhood. Final results from the wider study are currently being consolidated and analyzed.

\section{Conclusions}

Location and time are essential elements for designing compelling urban computing experiences that rely upon or exploit movement and space. The overlapping of space and narrative provides opportunities to design compelling experiences with a high level of immersion. However, designing such experiences generates many challenges: most of them not related to the technicalities of the application but rather to considerations of user context, behaviour, and perception.

In this paper we presented the design process, pilot and refinements of the interactive, location based Yasmine's Adventures Experience. The experience was designed in collaboration with the UDK design research group aiming to better integrate the Jewish Museum Berlin with the surrounding neighbourhood of Mehringplatz. We described our design process and the pilot evaluation of the first final prototype using the concept of flow. In flow state we lose track of time and anxiety and our level of focus maximizes our performance and pleasurable feelings coming from the activity.

Despite the cold weather and brevity of the pilot, insightful feedback was collected and important refinements were made to the application. The application was well received and further work is in progress to refine and disseminate more detailed knowledge in the area of location aware narratives and mobile storytelling applications for social benefit and community integration. Our users found Yasmine's Ad- 
ventures aesthetically pleasing and engaging. The mobile application was successful in inspiring people to learn more about the neighbourhood and the community's concerns. Location aware storytelling is one of the most compelling areas in the development of urban computing, but it also involves complex experiences that require further research in terms of methodology and evaluation criteria required for effective deployment.

\section{Acknowledgements}

This initiative was inspired by the Community Now? research project. We acknowledge the help of Bianca Herlo, who invited us to the symposium, and Iohanna Nicenboim from the UDK Design Research Group for giving us access to the content output of their community workshop 'Pinpointing Mehringplatz'. We wish to acknowledge the help of our fellow interns Rui Trindade and Paulo Bala and the support of the Associate Robotic Laboratories LARSyS (PEst-OE/EEI/LA0009/2013). The project has been developed as part of the Future Fabulators project (20131659/001-001 CU7 COOP7), funded by the EU Culture and Media program.

\section{$7 \quad$ References}

1. Paulos, E., Goodman, E.: The familiar stranger: anxiety, comfort, and play in public places. In Proceedings of the SIGCHI Conference on Human Factors in Computing Systems (CHI '04). pp.223-230. ACM, New York, NY, USA, (2004).

2. Berlin Jewish Museum Annual Report, 2011-2012, Museum Berlin (2013).

3. http://community-infrastructuring.org/pinpointing-mehringplatz/ (27/04/2015).

4. Stevens, Q. The Ludic City: Exploring the Potential of Public Spaces. Taylor \& Francis, 2007.

5. Reid, J:, Hull, R., Cater, K., Fleuriot, C., Magic Moments in situated mediascapes; in proceedings of the 2005 ACM SIGCHI International Conference in Advances in Computer Entertainment Technology, p.290-293, June 15-17, Valencia, Spain (2005)

6. Benford, S., Giannachi, G., Koleva, B., Rodden, T., From Interactions to Trajectories: Designing Coherent Journeys through Users Experiences, In Proc. CHI '09. April 4-9, Boston Massachusetts, USA, (2009).

7. Nisi, V., and Haahr, M. (2004). "Weirdview: Interactive Multilinear Narratives and RealLife Community Stories." Crossings: eJournal of Art and Technology 4(1).

8. Nisi, V., Oakley, I. \& Haahr, M. (2008), "Location-Aware Multimedia Stories: Bringing Together Real and Virtual Spaces". In Proceedings of ArTech 2008, Porto, Portugal.

9. Christopoulou, E.; Ringas, D.; Stefanidakis, M., "Experiences from the Urban Computing Impact on Urban Culture," Informatics (PCI), 2012 16th Panhellenic Conference on , vol., no., pp.56,61, 5-7 Oct. 2012 doi: 10.1109/PCi.2012.53.

10. Nisi, V., Oakley, I. \& PostHuma de Boer, M. (2010), "Locative narratives as Experiences: a new perspective on location aware multimedia stories". In Touchpoint Journal, Vol 1.3, 2009.

11. Dionisio, M., V. Nisi, and J. P. van Leeuwen, "The iLand of Madeira Location Aware Multimedia Stories", Interactive Storytelling, vol. 6432: Scottish Informat \& Comp Sci Alliance; EPSRC, Artificial Intelligence \& Games Res Network, pp. 147-152, 2010. 
12. O’Hara, K., Kindberg, T., Glancy, M., Baptista, L., Sukumaran, B., Kahana, G., and Rowbotham, J. Collecting and sharing location-based content on mobile phones in a zoo visitor experience. Comput. Supported Coop. Work 16, 1-2 (2007), 11-44.

13. Ciolfi, L., McLoughlin, M.: Designing for meaningful visitor engagement at a living history museum. In Proc. NordiCHI '2012, ACM, New York, NY, USA, 69-78 (2012).

14. Dionisio, M., "7 Stories: Location Based Story-Delivery System.", Master Thesis in Informatics and Engineering, CEEE, University of Madeira (UMA), Funchal, Portugal (2014).

15. Lochrie, M, Copic Pucihar, K, Gradinar, A \& Coulton, P 2013, 'Time-wARpXplorer: creating a playful experience in an urban time warp'. In Proceedings of Physical and Digital in Games and Play Seminar, Tampere, Finland, 29-30 May.

16. Čopic Pucihar, K., Coulton, P., Exploring the Evolution of Mobile Augmented Reality for Future Entertainment Systems. Under review for the Journal of Computers in Entertainment.

17. Olsson, T. (2012). User expectations and experiences of mobile augmented reality services. Tampereen teknillinen yliopisto. Julkaisu-Tampere University of Technology. Publication 1085.

18. Pietroni, E., "An augmented experiences in cultural heritage through mobile devices: "Matera tales of a city" project," Virtual Systems and Multimedia (VSMM), 2012 18th International Conference on , vol., no., pp.117,124, 2-5 Sept. 2012

19. Paul Dourish, Ken Anderson, and Dawn Nafus, Cultural Mobilities:Diversity and Agency in Urban Computing, Interact'2007,

20. Yu Zheng, Licia Capra, Ouri Wolfson, and Hai Yang. 2014. Urban computing: Concepts, methodologies and applications. ACM Trans. Intel. Sys. Tech. 5, 3, Art 38 (Sept 2014).

21. Csikszentmihalyi, M. (1990). Flow: The Psychology of Optimal Experience. NY Harper and Row.

22. Jenova Chen. 2007. Flow in games (and everything else). Commun. ACM 50, 4 (April 2007), 31-34

23. Magyaródi, T., Nagy, H., Soltész,P., Mózes,T., Oláh, A., Psychometric properties of a newly established flow state questionnaire, The Journal of Happiness \& Well-Being 2013, $1(2)$

24. Tuters, Marc, and Kazys Varnelis. "Beyond locative media: Giving shape to the internet of things." Leonardo 39.4 (2006): 357-363.

25. Nisi, Valentina; Ian Oakley and Mads Haahr (2008) "Location-Aware Multimedia Stories: Turning Spaces into Places" in Álvaro Barbosa (ed.) ARTECH 2008: Proceedings of the 4th International Conference on Digital Arts, Porto, 72-82.

26. Galloway, Anne; Ward, Matthew (July 2006). "Locative Media As Socialising And Spatializing Practice: Learning From Archaeology". Leonardo Electronic Almanac 14.3. 